\title{
Some Properties of Metrics in a Study on Convergence to Normality
}

\author{
C. C. HEYdE
}

Received January 17, 1968

Summary. This paper is concerned with the use of the $\mathscr{L}_{1}$ and $\mathscr{L}_{\infty}$ metrics in a study of certain properties and implications of convergence rates in the central limit theorem for sums of independent and identically distributed random variables which belong to the domain of attraction of the normal distribution. Also, some general convergence rate results on the $\mathscr{L}_{\infty}$ metric obtained under the assumption of a finite second moment are used as a vital tool in a new proof of the classical iterated logarithm law and in extending the scope of classical methods for the proof of other similar results of a more general kind.

\section{Introduction}

In this paper, we consider a sequence of independent and identically distributed random variables $X_{i}, i=1,2,3, \ldots$ which belong to the domain of attraction of the normal distribution. Since this last property implies $E\left|X_{i}\right|<\infty$, we shall suppose for convenience that $E X_{i}=0$. Then, if $S_{n}=\sum_{i=1}^{n} X_{i}, n \geqq 1$, there is a monotone sequence of normalizing constants $\left\{B_{n}, n \geqq 1\right\}$ such that as $n \rightarrow \infty$,

$$
F_{n}(x)=\operatorname{Pr}\left(S_{n} \leqq B_{n} x\right) \rightarrow \Phi(x)=\frac{1}{\sqrt{2 \pi}} \cdot \int_{-\infty}^{x} e^{-(1 / 2) u^{2}} d u
$$

and it is not difficult to see that as $n \rightarrow \infty$,

$$
\int_{-\infty}^{\infty}\left|F_{n}(x)-\Phi(x)\right| d x \rightarrow 0
$$

We shall firstly establish the following theorem:

Theorem 1. If

$$
\sum_{n=1}^{\infty} n^{-1} \int_{-\infty}^{\infty}\left|F_{n}(x)-\Phi(x)\right| d x<\infty
$$

then $E X_{i}^{2}<\infty$. That is, the $X_{i}$ belong to the domain of normal attraction of the normal distribution.

In connection with this theorem, we mention the results of IBRAGImov [7] where it is shown that if

$$
\int_{-\infty}^{\infty}\left|F_{n}(x)-\Phi(x)\right| d x=O\left(n^{-\eta}\right)
$$

for some $\eta>0$, then $E X_{i}^{2}<\infty$. Also, if $E X_{i}^{2}=\sigma^{2}<\infty$, then for $0<\delta<1$,

$$
\int_{-\infty}^{\infty}\left|\operatorname{Pr}\left(S_{n} \leqq x \sigma \sqrt{n}\right)-\Phi(x)\right| d x=O\left(n^{-\delta / 2}\right)
$$

13 Z. Wahrscheinlichkeitstheorie verw. Geb., Bd. 11 
if and only if, as $z \rightarrow \infty$,

$$
\int_{|x|>z} x^{2} d \operatorname{Pr}\left(X_{i} \leqq x\right)=O\left(z^{-\delta}\right)
$$

We shall show in Theorem 2 that if $E X_{i}^{2}=\sigma^{2}<\infty$, then

$$
\sum_{1}^{\infty} n^{-1} \int_{-\infty}^{\infty}\left|\operatorname{Pr}\left(S_{n} \leqq x \sigma \sqrt{n}\right)-\Phi(x)\right| d x<\infty
$$

if and only if $E X_{i}^{2} \log ^{+}\left|X_{i}\right|<\infty$. (Here, $\left.\log ^{+} x=\max (0, \log x)\right)$.

The other main theorem of this paper is Theorem 4. where

Theorem 4. Let $E X_{i}^{2}=1, F(x)=\operatorname{Pr}\left(X_{i} \leqq x\right)$, and $F_{n}(x)=\operatorname{Pr}\left(S_{n} \leqq x \sigma_{n} \sqrt{n}\right)$,

$$
\sigma_{n}^{2}=\int_{|x|<\sqrt{n}} x^{2} d F(x)-\left[\int_{|x|<\sqrt{n}} x d F(x)\right]^{2} .
$$

Then, if $K>0, C>1$, and $\left\{n_{k}, k \geqq 1\right\}$ is a sequence of integers with $n_{k} \sim K C^{2 k}$ as $k \rightarrow \infty$, we have

$$
\sum_{k=1}^{\infty} \sup _{x}\left|F_{n_{k}}(x)-\Phi(x)\right|<\infty
$$

This theorem depends on refinement of the details of proof of Theorem 1 of Friedman, Katz, and Koopmans [3] where it is not difficult to see that the following somewhat stronger result is, in effect, derived. then

Theorem A. If $E X_{i}^{2}=1$ and $F_{n}(x)=\operatorname{Pr}\left(S_{n} \leqq x \sigma_{n} \sqrt{n}\right)$ where $\sigma_{n}$ is given by (2),

$$
\sum_{n=1}^{\infty} n^{-1} \sup _{x}\left|F_{n}(x)-\Phi(x)\right|<\infty .
$$

We remark, in passing, that conditions (3) and (4) would, of course, be equivalent if it could be shown that the $L_{\infty}$ metric is ultimately monotonically convergent to zero. Theorem 4 is particularly useful from the point of view of applications since it is often convenient to study convergence along a geometric subsequence. We shall illustrate by obtaining a new proof of the law of the iterated logarithm and by showing how results of CHOver [1] may be extended.

\section{Proof of Theorem 1}

Let $F(x)=\operatorname{Pr}\left(X_{i} \leqq x\right)$, and suppose that $f(t)$ and $f_{n}(t)$ are the characteristic functions corresponding to $F(x)$ and $F_{n}(x)$ respectively. Then, integrating by parts in the equation

we obtain

$$
f_{n}(t)-e^{-\frac{1}{2} t^{2}}=\int_{-\infty}^{\infty} e^{i t x} d\left[F_{n}(x)-\Phi(x)\right]
$$

so that

$$
-\frac{f_{n}(t)-e^{-\frac{1}{2} t^{2}}}{i t}=\int_{-\infty}^{\infty} e^{i t x}\left[F_{n}(x)-\Phi(x)\right] d x
$$

$$
\left|\frac{f_{n}(t)-e^{-\frac{1}{2} t^{2}}}{t}\right| \leqq \int_{-\infty}^{\infty}\left|F_{n}(x)-\Phi(x)\right| d x
$$


and therefore using (1),

$$
\sum_{1}^{\infty} n^{-1}\left|\frac{f_{n}(t)-e^{-\frac{1}{2} t^{2}}}{t}\right|<\infty .
$$

Now, by Lemma 2.1 of [7], $f(t)$ is representable in the form

$$
f(t)=\exp \left\{-\frac{1}{2} t^{2} H(t)\right\}
$$

where $H(t)$ is a slowly varying function as $t \rightarrow 0$ and

as $t \rightarrow 0$. Then, from (6),

$$
H(t) \sim \int_{|x t| \leqq 1} x^{2} d F(x)
$$

$$
f_{n}(t)=\left[f\left(\frac{t}{B_{n}}\right)\right]^{n}=\exp \left\{-\frac{n t^{2}}{2 B_{n}^{2}} H\left(\frac{t}{B_{n}}\right)\right\}
$$

so that (5) gives for $t>0$,

$$
\sum_{1}^{\infty} n^{-1}\left|\exp \left\{-\frac{t^{2}}{2}\left[\frac{n}{B_{n}^{2}} H\left(\frac{t}{B_{n}}\right)-1\right]\right\}-1\right|<\infty
$$

However, as $n \rightarrow \infty$,

$$
\exp \left\{-\frac{t^{2}}{2}\left[\frac{n}{B_{n}^{2}} H\left(\frac{t}{B_{n}}\right)-1\right]\right\}-1=\frac{t^{2}}{2}\left[1-\frac{n}{B_{n}^{2}} H\left(\frac{t}{B_{n}}\right)\right](1+o(1))
$$

so that for $t>0$,

Therefore,

$$
\sum_{1}^{\infty} n^{-1}\left|1-\frac{n}{B_{n}^{2}} H\left(\frac{t}{B_{n}}\right)\right|<\infty
$$

$$
\begin{aligned}
\sum_{1}^{\infty} \frac{1}{B_{n}^{2-}} \mid H\left(\frac{1}{B_{n}}\right) & -H\left(\frac{2}{B_{n}}\right) \mid \\
& \leqq \sum_{1}^{\infty} n^{-1}\left|1-\frac{n}{B_{n}^{2}} H\left(\frac{1}{B_{n}}\right)\right|+\sum_{1}^{\infty} n^{-1}\left|1-\frac{n}{B_{n}^{2}} H\left(\frac{2}{B_{n}}\right)\right|<\infty
\end{aligned}
$$

or equivalently, in view of (6),

$$
\sum_{1}^{\infty}\left|\log f\left(2 B_{n}^{-1}\right)-4 \log f\left(B_{n}^{-1}\right)\right|<\infty \text {. }
$$

Now,

where

$$
\begin{aligned}
\log f\left(2 B_{n}^{-1}\right) & -4 \log f\left(B_{n}^{-1}\right) \\
& =\log \left[1-\left\{1-f\left(2 B_{n}^{-1}\right)\right\}\right]-4 \log \left[1-\left\{1-f\left(B_{n}^{-1}\right)\right\}\right] \\
& =-\left\{1-f\left(2 B_{n}^{-1}\right)\right\}+4\left\{1-f\left(B_{n}^{-1}\right)\right\}+A_{n},
\end{aligned}
$$

$$
A_{n}=\sum_{r=2}^{\infty} r^{-1}\left[4\left\{1-f\left(B_{n}^{-1}\right)\right\}^{r}-\left\{1-f\left(2 B_{n}^{-1}\right)\right\}^{r}\right] .
$$

Furthermore, as $n \rightarrow \infty$, we have

so that

$$
\begin{aligned}
1-f\left(2 B_{n}^{-1}\right) & \sim \frac{1}{2}\left(2 B_{n}^{-1}\right)^{2} H\left(2 B_{n}^{-1}\right) \sim 2 n^{-1}, \\
1-f\left(B_{n}^{-1}\right) & \sim \frac{1}{2} B_{n}^{-2} H\left(B_{n}^{-1}\right) \sim \frac{1}{2} n^{-1},
\end{aligned}
$$

$$
\sum_{1}^{\infty}\left|A_{n}\right|<\infty
$$


Consequently, from (7) and (8),

$$
\sum_{1}^{\infty}\left|4\left\{1-f\left(B_{n}^{-1}\right)\right\}-\left\{1-f\left(2 B_{n}^{-1}\right)\right\}\right|<\infty,
$$

and a fortiori, taking real parts,

$$
\sum_{1}^{\infty}\left|4\left\{1-\operatorname{Re} f\left(B_{n}^{-1}\right)\right\}-\left\{1-\operatorname{Re} f\left(2 B_{n}^{-1}\right)\right\}\right|<\infty .
$$

This may be rewritten as

$$
\sum_{1}^{\infty}\left|\int_{-\infty}^{\infty}\left\{4\left(1-\cos B_{n}^{-1} x\right)-\left(1-\cos 2 B_{n}^{-1} x\right)\right\} d F^{\prime}(x)\right|<\infty,
$$

which reduces to

$$
\sum_{1}^{\infty} \int_{-\infty}^{\infty}\left(1-\cos B_{n}^{-1} x\right)^{2} d F(x)<\infty
$$

Now, for $|x| \leqq \pi B_{n}$, we can find a positive constant $C$ so that

$$
1-\cos B_{n}^{-1} x \geqq C\left(B_{n}^{-1} x\right)^{2},
$$

and therefore, (9) yields

$$
\sum_{1}^{\infty} B_{n}^{-4} \int_{|x| \leqq \pi B_{n}} x^{4} d F(x)<\infty .
$$

Furthermore,

$$
\begin{aligned}
\sum_{n=1}^{\infty} B_{n}^{-4} \int_{|x| \leqq \pi B_{n}} x^{4} d F^{\prime}(x) & \geqq \sum_{n=2}^{\infty} B_{n}^{-4} \sum_{k=1}^{n-1} \int_{\pi B_{k}<|x| \leqq \pi B_{k+1}} x^{4} d F(x) \\
& \geqq \pi^{4} \sum_{n=2}^{\infty} B_{n}^{-4} \sum_{k=1}^{n-1} B_{k}^{4} \operatorname{Pr}\left(\pi B_{k}<\left|X_{i}\right| \leqq \pi B_{k+1}\right) \\
& =\pi^{4} \sum_{k=1}^{\infty} B_{k}^{4} \operatorname{Pr}\left(\pi B_{k}<\left|X_{i}\right| \leqq \pi B_{k+1}\right) \sum_{n=k+1}^{\infty} B_{n}^{-4}
\end{aligned}
$$

Also, $B_{n}=\sqrt{n} h(n)$ where $h(n)$ is slowly varying as $n \rightarrow \infty$ ([7], Lemma 2.2) so, using a standard result on regularly varying functions (e.g. FeLLER [2], II, 273), we have

$$
\sum_{n=k+1}^{\infty} B_{n}^{-4} \sim k B_{k}^{-4}
$$

as $k \rightarrow \infty$ and, from (10) and (11),

$$
\sum_{k=1}^{\infty} k \operatorname{Pr}\left(\pi B_{k}<\left|X_{i}\right| \leqq \pi B_{k+1}\right)<\infty
$$

But, since $B_{n}^{-1} S_{n}$ converges to normality, $n \operatorname{Pr}\left(\left|X_{i}\right|>\varepsilon B_{n}\right) \rightarrow 0$ as $n \rightarrow \infty$ for any $\varepsilon>0$ (e.g. Lòvve [8], 316), and therefore, from (12),

$$
\sum_{n=1}^{\infty} \operatorname{Pr}\left(\left|X_{i}\right|>\pi B_{n}\right)<\infty .
$$


Now suppose that $E\left|X_{i}\right|^{2}=\infty$, so that the slowly varying function

$$
K(t)=\int_{|x t| \leqq 1} x^{2} d F(x) \rightarrow \infty \text { as } t \rightarrow 0
$$

Under these circumstances, we shall obtain a contradiction with our assumption of convergence to normality by showing that the condition (13) implies that $B_{n}^{-1} S_{n}$ converges in probability to zero.

Using the degenerate convergence criterion (Lok̀ve [8], 317), we have $B_{n}^{-1} S_{n} \stackrel{p}{\rightarrow} 0$ provided that, as $n \rightarrow \infty$, and for any $\varepsilon>0$,

$$
n \operatorname{Pr}\left(\left|X_{i}\right|>\varepsilon B_{n}\right) \rightarrow 0, \frac{n}{B_{n}^{2-}} \int_{|x|<\pi B_{n}} x^{2} d F(x) \rightarrow 0 .
$$

As we have indicated, the former is satisfied so it remains to establish the latter. We have, since $n B_{n}^{-2} K\left(B_{n}^{-1}\right) \rightarrow 1$ as $n \rightarrow \infty$,

$$
\begin{aligned}
\int_{|x|<\pi B_{n}} x^{2} d F(x) & \leqq \int_{|x|<\pi B_{1}} x^{2} d F(x)+\pi^{2} \sum_{k=1}^{n} B_{k+1}^{2} \operatorname{Pr}\left(\pi B_{k}<\left|X_{i}\right| \leqq \pi B_{k+1}\right) \\
& \leqq \int_{|x|<\pi B_{1}} x^{2} d F(x)+C \sum_{k=1}^{n} K\left(B_{k}^{-1}\right) k \operatorname{Pr}\left(\pi B_{k}<\left|X_{i}\right| \leqq \pi B_{k+1}\right)
\end{aligned}
$$

for some positive constant $C$. Then, using (12) and by virtue of our assumptionthat $K(t) \rightarrow \infty$ as $t \rightarrow 0$, we have from the Kronecker Lemma (e.g. [8], 238), that

$$
\frac{n}{B_{n}^{2}} \int_{|x|<\pi B_{n}} x^{2} d F(x) \sim \frac{1}{K\left(B_{n}^{-1}\right)} \int_{|x|<\pi B_{n}} x^{2} d F(x) \rightarrow 0 \text { as } n \rightarrow \infty .
$$

This establishes the required contradiction. The result of the theorem is then immediate. (13) is only possible in the case where $K\left(B_{n}^{-1}\right)$ approaches a positive constant as $n \rightarrow \infty$ in which case it is easily seen that $E X_{i}^{2}<\infty$.

\section{Background to Theorem 1}

Theorem 2. Let $E X_{i}=0$ and $E X_{i}^{2}=\sigma^{2}<\infty$. Then, the following three conditions are equivalent:

(i) $\quad E X_{i}^{2} \log ^{+}\left|X_{i}\right|<\infty$,

(ii) $\quad \sum_{1}^{\infty} n^{-1} \int_{-\infty}^{\infty}\left|\operatorname{Pr}\left(S_{n} \leqq x \sigma \sqrt{n}\right)-\Phi(x)\right| d x<\infty$,

(iii) $\quad \sum_{1}^{\infty} n^{-1} \sup _{x}\left|\operatorname{Pr}\left(S_{n} \leqq x \sigma \sqrt{n}\right)-\Phi(x)\right|<\infty$.

Proof. The equivalence of (i) and (iii) has been established in HEyde [5]. We shall proceed to establish the equivalence of (i) and (ii).

Suppose firstly that (ii) holds. It is easily seen that the proof of the necessity part of the theorem of [5] is still applicable and (i) follows. If, on the other hand, (i) holds, we have from Lemma 2 of HEYDE [6] with $a_{n}^{2}=(2+\varepsilon) \log \log n$, $\varepsilon>0$, and $B_{n}^{2}=\sigma^{-2} \int x^{2} d \operatorname{Pr}\left(X_{i} \leqq x\right)$, that

$$
|x|<\sqrt{n}
$$

$$
\sum_{1}^{\infty} n^{-1} \int_{-\infty}^{\infty}\left|\operatorname{Pr}\left(S_{n} \leqq x \sigma \sqrt{n}\right)-\Phi\left(x B_{n}^{-1}\right)\right| d x<\infty
$$


if

(a) $\quad \sum_{1}^{\infty} n^{-1}\left(1-B_{n}^{2}\right)<\infty$

(b) $\quad \sum_{1}^{\infty} n^{-1} \int_{|x| \geqq a_{n}} x^{2} d \Phi\left(x B_{n}^{-1}\right)<\infty$,

(c) $\quad \sum_{3}^{\infty} n^{-1} \log \log n \sup _{x}\left|\operatorname{Pr}\left(S_{n} \leqq x \sigma \sqrt{n}\right)-\Phi\left(x B_{n}^{-1}\right)\right|<\infty$.

Furthermore, it is shown in the proof of the theorem of [6] that these three conditions are satisfied. Consequently, from (14), we see that (ii) holds if

$$
\sum_{1}^{\infty} n^{-1} \int_{-\infty}^{\infty}\left|\Phi\left(x B_{n}^{-1}\right)-\Phi(x)\right| d x<\infty
$$

Now,

so that

$$
\left|\Phi\left(x B_{n}^{-1}\right)-\Phi(x)\right|=\frac{1}{\sqrt{2 \pi}} \int_{|x|}^{|x| B_{n}^{-1}} e^{-\frac{1}{2} u^{2}} d u=\frac{|x|}{\sqrt{2 \pi}} \int_{1}^{B_{n}^{-1}} e^{-\frac{1}{2} u^{2} x^{2}} d u
$$

$$
\begin{aligned}
\int_{-\infty}^{\infty}\left|\Phi\left(x B_{n}^{-1}\right)-\Phi(x)\right| d x & =\frac{2}{\sqrt{2 \pi}} \int_{0}^{\infty} x\left\{\int_{1}^{B_{n}^{-1}} e^{-\frac{1}{2} u^{2} x^{2}} d u\right\} d x \\
& =\frac{2}{\sqrt{2 \pi}} \int_{1}^{B_{n}^{-1}}\left\{\int_{0}^{\infty} x e^{-\frac{1}{2} u^{2} x^{2}} d x\right\} d u \\
& =\frac{2}{\sqrt{2 \pi}} \int_{1}^{B_{n}^{-1}} u^{-2} d u \\
& =\frac{2}{\sqrt{2 \pi}}\left(1-B_{n}\right)<\frac{2}{\sqrt{2 \pi}}\left(1-B_{n}^{2}\right),
\end{aligned}
$$

and (15) holds in view of (a). This completes the proof of the theorem.

Next, we shall go on to examine the effect of the choice of $B_{n}$ on the magnitude of the $L_{1}$ metric $\int_{-\infty}^{\infty}\left|F_{n}(x)-\Phi(x)\right| d x$. In doing this we shall, in the light of Theorem 1, concentrate on the context of $E X_{i}^{2}=\sigma^{2}<\infty$, so that $B_{n}$ may be written in the form $B_{n}=\sigma \sqrt{n\left(1+\varepsilon_{n}\right)}$ where $\varepsilon_{n} \rightarrow 0$ as $n \rightarrow \infty$ ([7], Lemma 2.2).

As a starting point, we take a simple example which well illustrates the rôle of $\varepsilon_{n}$. Let the $X_{i}$ be normally distributed with unit variance. Then, since $S_{n} / \sqrt{n}$ has the same distribution as $X_{i}$ we have, as in (16),

$$
\int_{-\infty}^{\infty}\left|\operatorname{Pr}\left(S_{n} \leqq x \sqrt{n\left(1+\varepsilon_{n}\right)}\right)-\Phi(x)\right| d x=\int_{-\infty}^{\infty}\left|\Phi\left(x \sqrt{1+\varepsilon_{n}}\right)-\Phi(x)\right| d x
$$

so that we must have $\sum_{1}^{\infty} n^{-1}\left|\varepsilon_{n}\right|<\infty$ in order that

$$
\sum_{1}^{\infty} n^{-1} \int_{-\infty}^{\infty}\left|\operatorname{Pr}\left(S_{n} \leqq x \sqrt{n\left(1+\varepsilon_{n}\right)}\right)-\Phi(x)\right| d x<\infty
$$


With this kind of consideration in mind, we shall go on to establish the following theorem.

Theorem 3. Let $E X_{i}^{2} \log ^{+}\left|X_{i}\right|<\infty$ and write $B_{n}^{2}=n E X_{i}^{2}\left(1+\varepsilon_{n}\right)$ where $\varepsilon_{n} \rightarrow 0$ as $n \rightarrow \infty$. If $\sum_{1}^{\infty} n^{-1}\left|\varepsilon_{n}\right|<\infty$, then

$$
\sum_{1}^{\infty} n^{-1} \int_{-\infty}^{\infty}\left|\operatorname{Pr}\left(S_{n} \leqq B_{n} x\right)-\Phi(x)\right| d x<\infty .
$$

Conversely, if (18) holds for some monotone sequence $\left\{B_{n}\right\}$, and the $X_{i}$ belong to the domain of attraction of the normal distribution, then $E X_{i}^{2}<\infty$ and

$$
B_{n}^{2}=n E X_{i}^{2}\left(1+\varepsilon_{n}\right)
$$

where $\varepsilon_{n} \rightarrow 0$ as $n \rightarrow \infty$. If, in addition,

$$
\sum_{1}^{\infty} n^{-1}\left|\varepsilon_{n}\right|<\infty, \text { then } E X_{i}^{2} \log ^{+}\left|X_{i}\right|<\infty .
$$

Proof. Write $E X_{i}^{2}=\sigma^{2}(<\infty)$. We obtain from Theorem 2 that

$$
\sum_{1}^{\infty} n^{-1} \int_{-\infty}^{\infty}\left|\operatorname{Pr}\left(S_{n} \leqq x \sigma \sqrt{n}\right)-\Phi(x)\right| d x<\infty
$$

while

$$
\begin{aligned}
& \left|\operatorname{Pr}\left(S_{n} \leqq x \sigma \sqrt{n\left(1+\varepsilon_{n}\right)}\right)-\Phi(x)\right| \\
& \leqq\left|\operatorname{Pr}\left(S_{n} \leqq x \sqrt{\left(1+\varepsilon_{n}\right)} \sigma \sqrt{n}\right)-\Phi\left(x \sqrt{1+\varepsilon_{n}}\right)\right|+\left|\Phi\left(x \sqrt{1+\varepsilon_{n}}\right)-\Phi(x)\right|,
\end{aligned}
$$

so that the result of the first part of the theorem is immediate from (17) and (19).

Now suppose that (18) holds for some monotone sequence $\left\{B_{n}\right\}$, and the $X_{i}$ belong to the domain of attraction of the normal distribution. It follows from Theorem 1 that $E X_{i}^{2}=\sigma^{2}<\infty$, and consequently $B_{n}$ must be of the form $B_{n}=\sigma \sqrt{n\left(1+\varepsilon_{n}\right)}$ where $\varepsilon_{n} \rightarrow 0$ as $n \rightarrow \infty$. Further, the Eq. (20) can be rewritten to yield

$$
\int_{-\infty}^{\infty}\left|\operatorname{Pr}\left(S_{n} \leqq x \sigma \sqrt{n}\right)-\Phi(x)\right| d x \leqq \int_{-\infty}^{\infty}\left|\operatorname{Pr}\left(S_{n} \leqq x \sigma \sqrt{n\left(1+\varepsilon_{n}\right)}\right)-\Phi(x)\right| d x+C\left|\varepsilon_{n}\right|,
$$

where $C$ is a positive constant, so that, if $\sum_{1}^{\infty} n^{-1}\left|\varepsilon_{n}\right|<\infty$, (19) holds. The result $E X_{i}^{2} \log ^{+}\left|X_{i}\right|<\infty$ then follows from Theorem 2. This completes the proof.

\section{Proof of Theorem 4}

From the proof of Theorem 1 of Friedman, Katz, and Koopmans [3], we extract the information that

$$
\begin{aligned}
& n^{-1}\left|F_{n}\left(x \sigma_{n}^{-1}\right)-\Phi\left(x \sigma_{n}^{-1}\right)\right| \\
& \quad \leqq M n^{-3 / 2}\left\{a_{n}+\left|\mu_{n}\right|^{3}\right\}+n^{-1}\left|\Phi\left[\sigma_{n}^{-1}\left(x-\sqrt{n} \mu_{n}\right)\right]-\Phi\left(\sigma_{n}^{-1} x\right)\right|+c_{n},
\end{aligned}
$$

where $M$ is a positive constant,

$$
\mu_{n}=\int_{|x|<\sqrt{n}} x d F(x), \quad a_{n}=\int_{|x|<\sqrt{n}}|x|^{3} d F(x), \quad c_{n}=\operatorname{Pr}\left(\left|X_{i}\right|>\sqrt{n}\right),
$$


and also that $\sum_{n=1}^{\infty} n^{-3 / 2} a_{n}<\infty$, and $\sum_{n=1}^{\infty} c_{n}<\infty$. Now, since $E X_{i}=0$ and $E X_{i}^{2}=1$, it follows that $\sqrt{n} \mu_{n} \rightarrow 0$ and $1 \geqq \sigma_{n} \rightarrow 1$ as $n \rightarrow \infty$. Consequently, using the mean value theorem, we obtain

$$
\left|\Phi\left[\sigma_{n}^{-1}\left(x-\sqrt{n} \mu_{n}\right)\right]-\Phi\left(\sigma_{n}^{-1} x\right)\right| \leqq C \sqrt{n}\left|\mu_{n}\right|
$$

for some positive constant $C$ (independent of $x$ ). Furthermore, we note that $\mu_{n}=o(1)=o\left(a_{n}\right)$ as $n \rightarrow \infty$ and we can write instead of (21),

$$
n^{-1} \sup _{x}\left|F_{n}(x)-\Phi(x)\right| \leqq A n^{-3 / 2} a_{n}+B n^{-1 / 2} b_{n}+c_{n}
$$

where

$$
b_{n}=\int_{|x| \geqq \sqrt{n}}|x| d F(x) \geqq\left|\int_{|x| \geqq \sqrt{n}} x d F(x)\right|=\left|\mu_{n}\right|,
$$

and $A, B$ are positive constants.

Before proceeding, we check that the result (4) follows from (22) and to do this, in view of the abovementioned results, it is just necessary to show that $\sum_{n=1}^{\infty} n^{-1 / 2} b_{n}<\infty$. This is easily seen to be the case as $E X_{i}^{2}<\infty$, so that

$$
\begin{aligned}
\sum_{n=1}^{\infty} n^{-1 / 2} b_{n} & \leqq \sum_{n=1}^{\infty} n^{-1 / 2} \sum_{k=n}^{\infty}(k+1)^{1 / 2} \operatorname{Pr}\left(k \leqq X_{i}^{2}<k+1\right) \\
& =\sum_{k=1}^{\infty}(k+1)^{1 / 2} \operatorname{Pr}\left(k \leqq X_{i}^{2}<k+1\right) \sum_{n=1}^{k} n^{-1 / 2} \\
& \leqq C^{\prime} \sum_{k=1}^{\infty}(k+1) \operatorname{Pr}\left(k \leqq X_{i}^{2}<k+1\right)<\infty
\end{aligned}
$$

$C^{\prime}$ being a positive constant. We are now in a position to deduce (3) from (22) by showing that

$$
\sum_{k=1}^{\infty} n_{k}^{-1 / 2} a_{n_{k}}<\infty, \quad \sum_{k=1}^{\infty} n_{k}^{1 / 2} b_{n_{k}}<\infty \text { and } \sum_{k=1}^{\infty} n_{k} c_{n_{k}}<\infty .
$$

Since $a_{n}$ is non-decreasing in $n$,

$$
\sum_{n=1}^{\infty} n^{-3 / 2} a_{n} \geqq \sum_{k=1}^{\infty} \sum_{n=n_{k}}^{n_{k+1}-1} n^{-3 / 2} a_{n} \geqq \sum_{k=1}^{\infty}\left(n_{k+1}-n_{k}\right)\left(n_{k+1}-1\right)^{-3 / 2} a_{n_{k}} .
$$

However, since $n_{k} \sim K C^{2 k}$ as $k \rightarrow \infty$,

$$
\left(n_{k+1}-n_{k}\right)\left(n_{k+1}-1\right)^{-3 / 2} \sim C^{-3}\left(C^{2}-1\right) n_{k}^{-1 / 2},
$$

so that $\sum_{k=1}^{\infty} n_{k}^{-1 / 2} a_{n_{k}}<\infty$. Also, since $b_{n}$ is non-increasing in $n$,

$$
\sum_{n=1}^{\infty} n^{-1 / 2} b_{n} \geqq \sum_{k=1}^{\infty} \sum_{n=n_{k}+1}^{n_{k+1}} n^{-1 / 2} b_{n} \geqq \sum_{k=1}^{\infty} n_{k+1}^{-1 / 2}\left(n_{k+1}-n_{k}\right) b_{n_{k+1}}
$$

and

$$
n_{k+1}^{-1 / 2}\left(n_{k+1}-n_{k}\right) \sim n_{k+1}^{1 / 2}\left(1-C^{-2}\right)
$$


so that $\sum_{k=1}^{\infty} n_{k}^{1 / 2} b_{n_{k}}<\infty$. Finally, $c_{n}$ is non-increasing in $n$, so that

while

$$
\sum_{n=1}^{\infty} c_{n} \geqq \sum_{k=1}^{\infty} \sum_{n=n_{k}+1}^{n_{k+1}} c_{n} \geqq \sum_{k=1}^{\infty}\left(n_{k+1}-n_{k}\right) c_{n_{k+1}}
$$

$$
n_{k+1}-n_{k} \sim\left(1-C^{-2}\right) n_{k+1},
$$

and consequently, $\sum_{k=1}^{\infty} n_{k} c_{n_{k}}<\infty$. The result (3) then follows from (22).

Corollary 1. Let $E X_{i}^{2}=1$ and $\{\varphi(n), n \geqq 1\}$ be a monotone sequence. Then, if $K>0, C>1$, and $\left\{n_{k}, k \geqq 1\right\}$ is a sequence of integers with $n_{k} \sim K C^{2 k}$ as $k \rightarrow \infty$, the following two conditions are equivalent:

$$
\begin{aligned}
& \sum_{k=1}^{\infty} \operatorname{Pr}\left(S_{n_{k}}>\varphi\left(n_{k}\right) \sqrt{n_{k}}\right)<\infty \\
& \sum_{k=1}^{\infty}\left[\varphi\left(n_{k}\right)\right]^{-1} \exp \left\{-\frac{1}{2}\left[\varphi\left(n_{k}\right)\right]^{2} \sigma_{n_{k}}^{-2}\right\}<\infty,
\end{aligned}
$$

$\sigma_{n}$ being given by (2).

Proof. From Theorem 4, we immediately obtain the equivalence of (A) and the condition

$$
\sum_{k=1}^{\infty}\left[1-\Phi\left(\varphi\left(n_{k}\right) \sigma_{n_{k}}^{-1}\right)\right]<\infty .
$$

The result of the corollary then follows since, as $k \rightarrow \infty$,

$$
\begin{aligned}
1-\Phi\left(\varphi\left(n_{k}\right) \sigma_{n_{k}}^{-1}\right) & \sim \frac{1}{\sqrt{2 \pi}} \frac{\sigma_{n_{k}}}{\varphi\left(n_{k}\right)} \exp \left\{-\frac{1}{2}\left[\varphi\left(n_{k}\right)\right]^{2} \sigma_{n_{k}}^{-2}\right\} \\
& \sim \frac{1}{\sqrt{2 \pi}} \frac{1}{\varphi\left(n_{k}\right)} \exp \left\{-\frac{1}{2}\left[\varphi\left(n_{k}\right)\right]^{2} \sigma_{n_{k}}^{-2}\right\}
\end{aligned}
$$

using a well-known asymptotic formula (e.g. FeLLer [2], I, 166).

\section{Applications of Theorem 4}

In this section, we shall illustrate the usefullness of Theorem 4 and Corollary 1 with two applications. We start by giving a new proof of the classical iterated logarithm law based on the use of Corollary 1. By making use of the corollary, we are able to avoid the ordinary complications of truncation and the use of exponential bounds.

Theorem 5 (HARTMAN and Wintner [4]). If $E X_{i}=0$ and $E X_{i}^{2}=1$, then

$$
\operatorname{Pr}\left(\limsup _{n \rightarrow \infty} \frac{S_{n}}{\sqrt{ } 2 n \log \log n}=1\right)=1 \text {. }
$$

Proof. Let $b_{n}=(2 n \log \log n)^{1 / 2}$ and $t_{n}=(2 \log \log n)^{1 / 2}$. Firstly we shall show that if $\delta>0$, then $\operatorname{Pr}\left(S_{n}>(1+\delta) b_{n}\right.$ i.o.) $=0$ ("i.o." stands for "infinitely often"). 
For $c>1$, let $n_{k}$ be the integer part of $c^{2 k}, k \geqq 1$. Then, if $M_{n}=\max S_{k}$, we have

where

$$
\operatorname{Pr}\left(S_{n}>(1+\delta) b_{n} \text { i.o. }\right) \leqq \operatorname{Pr}\left(M_{n_{k}}>(1+\delta) b_{n_{k-1}} \text { i.o. }\right)
$$

$$
(1+\delta) b_{n_{k-1}} \sim c^{-1}(1+\delta) b_{n_{k}} .
$$

Thus, taking $\delta^{\prime}$ with $0<\delta^{\prime}<\delta$, we can select $c>1$, so that $c^{-1}(1+\delta)>1+\delta^{\prime}$, and then,

$$
\operatorname{Pr}\left(M_{n_{k}}>(1+\delta) b_{n_{k-1}} \text { i.o. }\right) \leqq \operatorname{Pr}\left(M_{n_{k}}>\left(1+\delta^{\prime}\right) b_{n_{k}} \text { i.o. }\right) .
$$

Consequently, the result $\operatorname{Pr}\left(S_{n}>(1+\delta) b_{n}\right.$ i.o. $)=0$ will follow from the BorelCantelli lemma if we show that

$$
\sum_{k=1}^{\infty} \operatorname{Pr}\left(M_{n_{k}}>\left(1+\delta^{\prime}\right) b_{n_{k}}\right)<\infty .
$$

Now, using an inequality due to Kolmogorov (e.g. Lò̀ve [8], 248),

$$
\begin{aligned}
\operatorname{Pr}\left(M_{n_{k}}>\left(1+\delta^{\prime}\right) b_{n_{k}}\right) & \leqq 2 \operatorname{Pr}\left[S_{n_{k}}>\left(1+\delta^{\prime}-t_{n_{k}}^{-1} \sqrt{2}\right) b_{n_{k}}\right] \\
& \leqq 2 \operatorname{Pr}\left(S_{n_{k}}>\left(1+\delta^{\prime \prime}\right) b_{n_{k}}\right)
\end{aligned}
$$

for $0<\delta^{\prime \prime}<\delta^{\prime}$ and $k$ sufficiently large. Thus, by appeal to Corollary 1, we have convergence in $(23)$ if

$$
\sum_{k=1}^{\infty} t_{n_{k}}^{-1} \exp \left\{-\frac{1}{2}\left(1+\delta^{\prime \prime}\right)^{2} t_{n_{k}}^{2} \sigma_{n_{k}}^{-2}\right\}<\infty .
$$

This is easily seen to be the case as

$$
\begin{aligned}
t_{n_{k}}^{-1} \exp \left\{-\frac{1}{2}\left(1+\delta^{\prime \prime}\right)^{2} t_{n_{k}}^{2} \sigma_{n_{k}}^{-2}\right\} & \leqq t_{n_{k}}^{-1} \exp \left\{-\frac{1}{2}\left(1+\delta^{\prime \prime}\right) t_{n_{k}}^{2}\right\} \\
& =O\left(k^{-\left(1+\delta^{\prime \prime}\right)}(\log k)^{-1 / 2}\right) \text { as } \quad k \rightarrow \infty
\end{aligned}
$$

In order to complete the proof of the theorem it remains to show that

$$
\operatorname{Pr}\left(S_{n}>(1-\delta) b_{n} \text { i.o. }\right)=1 \quad \text { for } \delta>0 .
$$

Take $1>\delta>\delta^{\prime}>0$ and let

and

$$
\begin{aligned}
& u_{k}^{2}=n_{k}-n_{k-1} \sim n_{k}\left(1-c^{-2}\right), \\
& v_{k}=\left(2 \log \log u_{k}^{2}\right)^{1 / 2} \sim\left(2 \log \log n_{k}\right)^{1 / 2}=t_{n_{k}},
\end{aligned}
$$

Then,

$$
A_{k}=\left\{S_{n_{k}}-S_{n_{k-1}}>\left(1-\delta^{\prime}\right) u_{k} v_{k}\right\}
$$

$$
\operatorname{Pr}\left(A_{k}\right)=\operatorname{Pr}\left(S_{n_{k}-n_{k-1}}>\left(1-\delta^{\prime}\right) u_{k} v_{k}\right),
$$

while for $1>\delta^{\prime}>\delta^{\prime \prime}>0$ and $k$ sufficiently large,

$$
\begin{aligned}
v_{k}^{-1} \exp \left[-\frac{1}{2}\left(1-\delta^{\prime}\right)^{2} v_{k}^{2} \sigma_{n_{k}-n_{k-1}}^{-2}\right] & \geqq v_{k}^{-1} \exp \left[-\frac{1}{2}\left(1-\delta^{\prime \prime}\right)^{2} v_{k}^{2}\right] \\
& =O\left(k^{-\left(1-\delta^{\prime \prime}\right)^{2}}(\log k)^{-1 / 2}\right),
\end{aligned}
$$

so that from Corollary $1, \sum_{k=1}^{\infty} \operatorname{Pr}\left(A_{k}\right)=\infty$. Therefore, since the sums $S_{n_{k}}-S_{n_{k-1}}$ are non-overlapping, we have from the Borel-Cantelli lemma that $\operatorname{Pr}\left(A_{k}\right.$ i.o. $)=1$. Furthermore, if $B_{k}=\left\{\left|S_{n_{k-1}}\right| \leqq 2 b_{n_{k-1}}\right\}$, it follows from the first part of the 
proof that $\operatorname{Pr}\left(\bar{B}_{k}\right.$ i.o. $)=0$, where we have used a bar to denote the complementary event. We must therefore have $\operatorname{Pr}\left(A_{k} \cap B_{k}\right.$ i.o. $)=1$. Also,

while

$$
A_{k} \cap B_{k} \subset\left\{S_{n_{k}}>\left(1-\delta^{\prime}\right) u_{k} v_{k}-2 b_{n_{k-1}}\right\},
$$

$$
\left(1-\delta^{\prime}\right) u_{k} v_{k}-2 b_{n_{k-1}} \sim\left[\left(1-\delta^{\prime}\right)\left(1-c^{-2}\right)^{1 / 2}-2 c^{-1}\right] b_{n_{k}},
$$

so that if we take $c$ so large that

we have

$$
\left(1-\delta^{\prime}\right)\left(1-c^{-2}\right)^{1 / 2}-2 c^{-1}>1-\delta,
$$

$$
1==\operatorname{Pr}\left(A_{k} \cap B_{k} \text { i.o. }\right) \leqq \operatorname{Pr}\left(S_{n_{k}}>(1-\delta) b_{n_{k}} \text { i.o. }\right) .
$$

This implies, a fortiori, that $\operatorname{Pr}\left(S_{n}>(1-\delta) b_{n}\right.$ i.o. $)=1$ and thus completes the proof. We next proceed to our final application.

In the paper [9], STRASSEN obtained a deep and rather striking generalization of the iterated logarithm law. For his proof, he appealed to a result of Skorokhod which permits one to realize a sequence of independent and identically distributed random variables with finite variance in terms of random increments of a Brownian motion process. Later, CHOver [1] developed a more classical approach to the same results but was forced to introduce an extra moment condition $E\left|X_{i}\right|^{2+\delta}<\infty$, some $\delta>0$, in order to use an estimate of Esseen on convergence to normality. As we shall indicate here, Theorem 4 provides just the tool necessary to extend Chover's proof to obtain the results given by Strassen (the $\delta=0$ case). The reader is referred to the papers [1] and [9] for notations and explainations.

The are three places in the paper [1] where the suplementary moment condition $E\left|X_{i}\right|^{2+\delta}<\infty$, some $\delta>0$, is required. In each case it may be avoided by use of Theorem 4 (or Corollary 1). The applications are routine in character so we shall only carry out one of them (the last).

We need to show (see equations 30 and 32 of [1]) that for fixed $v$,

where

$$
\sum_{r} \operatorname{Pr}\left(\left|Z_{r}-A\right|<(2 m)^{-1} \varepsilon\right)=\infty,
$$

But,

$$
Z_{r}=\left(2 m N_{r, v} \log \log n_{r}\right)^{-1 / 2} S_{N_{r, v},} \quad A=\left[g\left(\frac{v+1}{m}\right)-g\left(\frac{v}{m}\right)\right] .
$$

$$
\operatorname{Pr}\left(\left|Z_{r}-A\right|<(2 m)^{-1} \varepsilon\right)=\operatorname{Pr}\left(Z_{r}<A+(2 m)^{-1} \varepsilon\right)-\operatorname{Pr}\left(Z_{r} \leqq A-(2 m)^{-1} \varepsilon\right),
$$

and by Theorem 4, since $N_{r, v} \sim m^{r-1}$,

$$
\sum_{r}\left|\operatorname{Pr}\left(Z_{r}<A+(2 m)^{-1} \varepsilon\right)-\operatorname{Pr}\left(Z_{r} \leqq A-(2 m)^{-1} \varepsilon\right)-\Phi(a+b)+\Phi(a-b)\right|<\infty
$$

where $s=\sigma_{N_{r, v}} \rightarrow 1$ as $r \rightarrow \infty$,

$$
a=A\left(2 m \log \log n_{r}\right)^{1 / 2} s^{-1}, \quad \text { and } \quad b=(2 m)^{-1} \varepsilon\left(2 m \log \log n_{r}\right)^{1 / 2} s^{-1} .
$$

Therefore, (24) holds provided that

$$
\sum_{r}|\Phi(a+b)-\Phi(a-b)|=\infty
$$

If $A=0$, this is easily seen to be the case since

$$
\Phi(b)-\Phi(-b)=2 \Phi(b)-1 \rightarrow 1
$$


as $r \rightarrow \infty$. Now suppose $A \neq 0$ and that $\varepsilon$ is small compared with $A$. Then, for $r$ sufficiently large,

$$
\begin{aligned}
\Phi(a+b)-\Phi(a-b)= & \Phi(|a|+b)-\Phi(|a|-b) \\
\sim & 1-\Phi(|a|-b) \\
\sim & \left(4 \pi m \log \log n_{r}\right)^{-1 / 2}\left(|A|-(2 m)^{-1} \varepsilon\right)^{-1} \\
& \cdot \exp \left\{-\left(|A|-(2 m)^{-1} \varepsilon\right)^{2} m s^{-2} \log \log n_{r}\right\} \\
\geqq & \left(4 \pi m \log \log n_{r}\right)^{-1 / 2}\left(|A|-(2 m)^{-1} \varepsilon\right)^{-1} \\
& \cdot \exp \left\{-A^{2} m \log \log n_{r}\right\} \\
= & O\left(r^{-A^{2} m}(\log r)^{-1 / 2}\right)
\end{aligned}
$$

as $r \rightarrow \infty$ since $n_{r} \sim m^{r}$. Also,

$$
\begin{aligned}
A^{2}=\left[g\left(\frac{v+1}{m}\right)-g\left(\frac{v}{m}\right)\right]^{2} & =\left[\int_{m^{-1} v}^{m^{-1}(v+1)} \dot{g}(t) d t\right]^{2} \\
& \leqq \int_{m^{-1} \nu}^{m^{-1}(v+1)} d t \int_{m^{-1} \nu}^{m^{-1}(v+1)}[\dot{g}(t)]^{2} d t \leqq m^{-1}
\end{aligned}
$$

s) that from (26) we see that (25) holds and the required result is obtained.

\section{References}

1. Chover, J.: On Strassen's version of the $\log \log$ law. Z. Wahrscheinlichkeitstheorie verw. Geb. 8, 83-90 (1967).

2. Feller, W.: An introduction to probability theory and its applications, Vols I, II. New York: Wiley 1957, 1966.

3. Friedman, N., M. Katz, and L. H. Koopmans: Convergence rates for the central limit theorem. Proc. nat. Acad. Sci. U.S.A. 56, 1062-1065 (1966).

4. Hartman, P., and A. Wintner: On the law of the iterated logarithm. Amer. J. Math. 63, $169-176(1941)$.

5. Heyde, C. C.: On the influence of moments on the rate of convergence to the normal distribution. Z. Wahrscheinlichkeitstheorie verw. Geb. 8, 12-18 (1967).

6. - On the growth of a random walk. Ann. Inst. Stat. Math. 20, 315-321 (1968).

7. Ibragimov, I. A.: On the accuracy of the Gaussian approximation to the distribution function of sums of independent variables. Theor. Probab. Appl. 11, 559-576 (1966).

8. Loغ̀ve, M.: Probability theory (3rd edition). New York: Van Nostrand 1963.

9. Strassen, V.: An invariance principle for the law of the iterated logarithm. Z. Wahrscheinlichkeitstheorie verw. Geb. 3, 211-226 (1964).

\section{Dr. C. C. Heyde}

Department of Statistics

Australian Natural University,

School of General Studies,

Box 4, P. O.,

Canberra, A. C. T. 2600,

Australia 